Journal of Methods and Measurement in the Social Sciences

Volume 1, Issue 2 (2010)

\title{
Editorial Personnel
}

\section{Editor}

Melinda F. Davis, University of Arizona, AZ, USA

\section{Production Editors}

J . Michael Menke, Critical Path Institute, AZ, USA

Mei-kuang Chen, University of Arizona, AZ, USA

\section{Executive Editorial Board}

Leonard Bickman, Vanderbilt University, TN, USA

Richard Bootzin, University of Arizona, AZ, USA

Richard Gorsuch, Fuller Theological Seminary, CA, USA

Lee Sechrest, University of Arizona, AZ, USA

Will Shadish, University of California, Merced, CA, USA

Souraya Sidani, University of Ryerson, Toronto, CA

David Weiss, University of Minnesota, MN, USA 